\title{
3D Technology as an Effective Tool for Reflection Simulation: The Beagle 2 lander on Mars
}

\author{
Teodora Kuzmanova \\ Imaging and Displays Research Group \\ De Montfort University \\ Leicester LE19BH, UK \\ teodora.kuzmanova@dmu.ac.uk
}

\author{
Nick Higgett \\ Digital Design Group, School of Design \\ De Montfort University \\ Leicester LE1 9BH, UK \\ nph@dmu.ac.uk
}

\author{
Mark Sims \\ Astrobiology and Space Instrumentation \\ University of Leicester \\ Leicester LE1 7RH, UK \\ mrs@le.ac.uk
}

\author{
Jim Clemmet \\ Beagle 2 Chief Engineer (retired) \\ UK \\ jim.clemmet@nt/world.com
}

\author{
Eric Tatham \\ Digital Design Group, School of Design \\ De Montfort University \\ Leicester LE1 9BH, UK \\ nph@dmu.ac.uk
}

\begin{abstract}
Beagle 2, developed for the European Space Agency's Mars Express Mission by the Beagle 2 Consortium, was due to land on Mars on December 25th, 2003. After being successfully ejected from the ESA's orbiter Mars Express, followed by an attempted landing, the spacecraft failed to communicate with Earth, and the mission was presumed lost. However, in January 2015, it was announced that satellite images from NASA's Mars Reconnaissance Orbiter (MRO) indicated the possibility of a successful landing by Beagle 2. In the light of these findings and the considerable uncertainty still surrounding the outcome of the mission, a team of researchers from De Montfort University and the University of Leicester have joined in a collaborative project, which aimed at identifying if the object, captured by NASA's HiRISE camera is Beagle 2, and detecting its possible landing configuration. The practical scientific experiment employed the innovative concept of 'reflection analysis', propound by Dr Mark Sims - former Beagle 2 Mission Manager and Professor of Astrobiology and Space Instrumentation at the Space Research Centre, University of Leicester. The technique stemmed from the idea of simulating possible configurations of the Beagle 2 lander, testing how they reflect light, and comparing the 3D renders to unprocessed images, available from the MRO's HiRISE camera at a number of different sun angles. The De Montfort University's team used commercial 3D modelling technology to create a 3D model of the spacecraft and replicate virtually the sun angles at the times the satellite images were taken. This allowed a comparison of the simulated 3D renders to the satellite images in order to estimate the configuration of Beagle 2 on Mars. The results revealed that Beagle 2 probably deployed at least three, and possibly all four of its solar panels after landing on the planet's surface.
\end{abstract}

3D simulation. Virtual reconstruction. Reflection analysis. Image correlation.

\section{INTRODUCTION}

The Finding of Beagle 2 project began in 2015, twelve years after the loss of signal from the lander, and stemmed from the discovery of an ostensibly artificial object near the intended Beagle 2 landing site on Mars, captured on satellite images from NASA's Mars Reconnaissance Orbiter instrument HiRISE.
The scientific collaboration between De Montfort University and the University of Leicester was aimed at an extensive experimentation with 3D modelling and imaging software to recreate the UK Mars spacecraft Beagle 2 in a range of landing configurations to determine whether a virtual simulation could confirm if the current Mars satellite images are actually of Beagle 2 and further determine its surface configuration. The project was carried out at the instigation and with the expert assistance of Professor Mark Sims, from the 
Space Research Centre at University of Leicester, who was the mission manager of the original Beagle 2 team.

This paper details the technological process of the $3 \mathrm{D}$ reconstruction of Beagle 2, the simulation of sun angles using a virtual camera, and outlines the image correlation process used for the comparison between the simulation and satellite images. It also describes the results achieved, and the scientific conclusions they led to.

\section{BACKGROUND}

Beagle 2 was a lander, developed for the European Space Agency's Mars Express Mission by the Beagle 2 Consortium, which was led by the Open University, headed by the late Professor Colin Pillinger. It was Europe's first Mars Lander project, and the paramount objective of its mission was to land onto the surface of Mars in search for signs of life.

Beagle 2 was part of the ESA's Mars Express Mission launched in June 2003. All pre-ejection telemetry demonstrated the probe was functioning adequately, and it was successfully ejected from the Mars Express spacecraft on the $19^{\text {th }}$ December 2003. The lander's entry into the atmosphere of Mars was due six days later, on 25th December, and the first data transmission from the surface of the red planet was expected just a few hours after successful deployment of Beagle 2's solar panels, and the uncovering of the communication antenna. Despite all expectations, no signal was received, and the probe was presumed lost until late 2014.

This was until nearly a decade later when images taken by NASA's Mars Reconnaissance Orbiter (MRO) shed new light on the mystery of the mission's fate, by indicating the possibility of a successful landing of Beagle 2. Despite its detection on the surface of Mars, due to the small size of the lander and the resolution of the HiRISE camera on the MRO, it was not clear whether the recorded object is in fact Beagle 2, and if so, what is the exact configuration of the lander on Mars.

In the light of these findings and the considerable uncertainty still surrounding the outcome of the mission, a team of researchers from De Montfort University and the University of Leicester, including the author of this paper, joined in a collaborative project, which hoped to identify if the object, captured by NASA's HiRISE camera was Beagle 2, as well as detect its possible landing configuration. The interdisciplinary nature of the project was the precursor of the alliance between De Montfort University's team of digital designers and the University of Leicester's space scientists.
The practical experiment proposed the employment of the innovative concept of 'reflection analysis', propound by Dr Mark Sims - former Beagle 2 Mission Manager. The technique stemmed from the idea of simulating possible configurations of the Beagle 2 lander, testing how they reflect light, and comparing the $3 \mathrm{D}$ renders to the unprocessed images, available from the MRO's HiRISE camera at a number of different sun angles.

The collaboration between De Montfort University and the University of Leicester used 3D modelling technology to create a basic 3D model of the spacecraft and replicate virtually the sun angles at the times the satellite images were taken. This would allow the matching of the simulated 3D renders to the real satellite images in order to estimate the deployed configuration of Beagle 2 on Mars.



Figure 1: Beagle 2 landing site, UK Space Agency

\section{PROJECT FOUNDATION}

The project started with the collection of information about the lander, its comprising elements, their exact purpose and function. A secondary investigation of relevant literature in the field of planetary research and the Beagle 2 experiment as a "concept instrument package" and "lander design study" (Pillinger 2003) was carried out, followed by a synthesis and evaluation of the gathered information with respect to the proposed reconstruction. The formation of this in-depth understanding of its operation as a mechanical system facilitated the consecutive virtual reconstruction of the probe and the simulation processes within the 3D software.

The project proceeded with an investigation of the technological characteristics of Autodesk Maya and its applications through different sources of information, such as theoretical literature, practical manuals, web resources and video tutorials. The literature review was followed by practical experimentation with the program, which is largely 
used in industry for modelling, animation and VFX. The project explored Autodesk Maya's potential for an accurate reconstruction of physical objects and simulation of lighting and dynamics. This research also aimed at identifying 3D elements, nodes, settings and processes, which would determine an efficient workflow for a detailed and precise recreation of Beagle 2.

The initial experimentation with the software was aimed at providing evidence for the level of precision, offered by creative 3D software for the reconstruction of physical devices and equipment, as well as the software's suitability for virtual simulation, and recognised its viability for the development of the novel research technique of 'reflection analysis'.

Investigation of the opportunities presented by the use of Physical Sky in combination with mia_exposure nodes, Indirect Lighting options and Tone Mapper, established Maya's suitability and functionality for a simulation of the lighting conditions on Mars and the particular sun angles data, recorded by the MRO's HiRISE camera. Testing the level of accuracy, achievable with the use of the Mental Ray renderer and mia materials characteristics, proved a high level of realism regarding the reflectivity of the lander's surfaces, essential for subsequent adequate comparison to the images, captured by the HiRISE camera. The practical use of Autodesk Maya concluded with analysis and assessment of the advantages and disadvantages of virtual cameras in terms of resolution and capability to simulate specific physical distance, and their application for the generation of final image renders. This initial stage demonstrated the software's potential to be used for the reflection simulation.



Figure 2: Final 3D render of the Beagle 2 lander

\section{DEVELOPMENT PROCESS}

\subsection{Modelling}

The 3D reconstruction of the Beagle 2 model was based on background information provided by Professor Mark Sims, which included dimensions of the lander. The initial objects used for the modelling of the lander and its lid were Polygon Primitives, as they would allow greater precision in the construction of the shapes. The Distance Tool was applied for creating accurate dimensions for the 3D asset.

All elements of the lander were modelled separately and grouped together. It was established that the most effective approach to the virtual reconstruction of the base and the lid shape should use low poly meshes (or, in other words objects with a low poly count), which were then exponentially smoothed and further refined to match the physical probe's shape as accurately as possible.

The solar panels' base and the glass surface covering them used a STEP file - a 3D model file formatted in STEP (Standard for the Exchange of Product Data), which was provided by engineers at the Leicester University, and ensured the necessary precision of the panels' shape and dimensions.

The modelling of the solar arrays utilised a basic Polygon Cube, scaled and duplicated to match the exact number and layout configuration of the arrays of the probe. The Duplicate Special edit option was used to ensure an accurate repetition of the objects and the even spacing between them. All duplicates were then combined in a single object to decrease the number of comprising elements and ensure more effective animation. The modelling of the robotic arm used as a reference a computer aided design schematic of the of the deployed Beagle 2 lander (Pillinger, p.80). The heat shield was also modelled for further accuracy of the virtual simulation and subsequent comparison to HiRISE images. The Sculpt Geometry Tool was used to simulate the topology of the surface of Mars from a Polygon Plane. The dialogue with the Leicester University's space scientists established that the greatest differences in the terrain of the lander's immediate vicinity should not exceed a height corresponding to $5-10 \mathrm{~cm}$.

\subsection{Animation}

After the creation of the 3D model and the final modifications of its shape, aimed at achieving a precise, even though simplified replica of Beagle 2, the model was rigged in order to animate a sequence of opening of the solar panels and the robotic arm. Despite of the project's ultimate aim being accurate image correlation and analysis of the pairs of simulated stills and HiRISE data, the animation played a crucial part in the accuracy of the results. 
In the initial stages of development of the practical work it was established that animation would be instrumental in terms of sequencing panel deployment in quick succession and precision of deployment degrees. Adjusting the rotation attributes of the virtual panels manually in order to simulate their deployment at a particular angle would have been a highly inefficient approach, as the operation would have to be repeated prior to taking every single render. The manual regulation would have lacked the same level of precision and proven to be much more time consuming, due to the great number of test renders, required by the experiment.

The animation technique, which was established as most effective, employed the use of Driven Keys. Driven keys "create an association between pairs of attributes, where one attribute value (or multiple attributes values) drives the value of another" (Autodesk 2016). The greatest benefit of this approach to animation is that after the relationship between attributes has been established, the Driven attribute does not have to be animated separately - its changes occur automatically as a result of a manipulation of the Driver object.

For the purpose of animating the probe, a fully transparent sphere and four cylinders were assigned to drive the opening of the lander's lid and the deployment of solar panels. The change of the translation value of the sphere along the $Y$ axis was set to be responsible for the rotation of the lid and the change of the translation values of the cylinders, each of which would then in turn drive a particular solar panel's rotation. The movement of the sphere as a main controller was keyframed along the Time Slider, so that the opening of the lander's lid and the deployment of each panel would occur in a timed, pre-set sequence, matching completely the opening sequence of the physical probe.

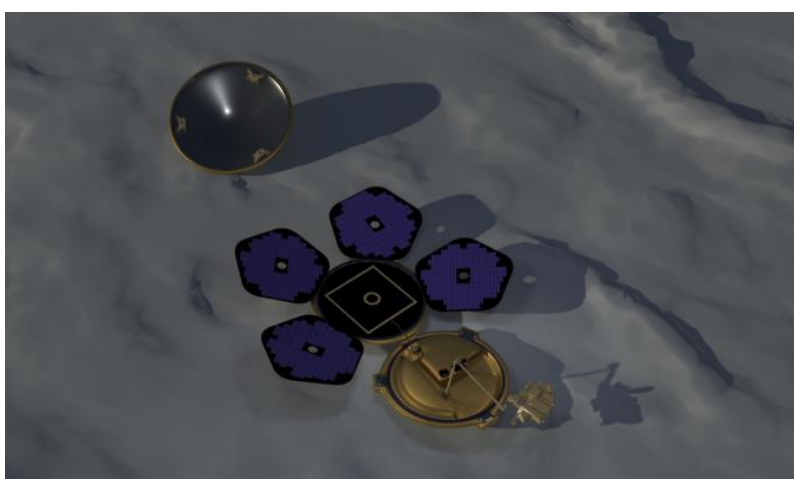

Figure 3: Lander with four panels and robotic arm deployed, heat shield and terrain (WIP render)

Finally, the opening of the robotic arm was also linked to the main controller, even though this specific part of the animation utilised a slightly different approach. As setting the change in the Translate $Y$ attribute of the controller to drive the displacement in space of all the comprising parts of the arm while keeping its integrity intact would have been a nearly impossible task, the robotic arm of the probe was rigged with the use of a joint structure. The Translate and Rotate attributes of the top node of the joint hierarchy were then linked to a controller, which in turn was set to be driven by the main controller.

\subsection{Texturing and lighting}

There was an in-depth experimentation with MIA materials (available through the Mental Ray plug-in for Autodesk Maya), aimed at assigning to the 3D model materials, which features were as close to the characteristics of the physical materials used for the creation of Beagle 2, as possible.

All MIA materials include Hardware Texturing attributes, which makes it possible for them to be previewed in the scene view. They are most commonly employed to simulate materials used in architectural and product design renderings and create a "physically accurate representation of hard-surface materials such as metal, wood and glass" (Autodesk 2014).

The decision to use MIA materials was reached due to the physical characteristics of the materials used for the construction of Beagle 2 - carbon fibre and shiny resin, and real gold for the outer surface of the lander, aluminium for the inner part of the shield and a glass plate, covering the solar panels. The effectiveness of the Mental Ray materials was established further by their support of anisotropic reflections and refractions, in-built indirect illumination controls, ability to create more natural and photorealistic rendering outcomes and advanced features for glossy reflections, emulated glossiness and importance sampling for enhanced performance, thus reducing rendering times. Another important feature of MIA shader, which was necessary for achieving the realistic look of the employed materials, was BRDF (Bidirectional Reflectance Distribution Function), where the reflectivity of a surface is dependent on the view angle. This was vital as the reflection analysis relies on the accuracy of the reflected light angles.

The attribute values for the specific materials were determined by a series of experiments, taking in the physical characteristics of the materials used for the construction of the probe and comparing the intensity of reflected light from test renders to images from the MRO's HiRISE camera.

The lighting conditions on Mars and the data for sun angles provided the background information for the project. These were replicated within the Maya 
software using Physical Sun and Sky and the Photographic Exposure node. Global Illumination and Caustics were used for the creation of more realistically accurate lighting, reflections and refraction of light.

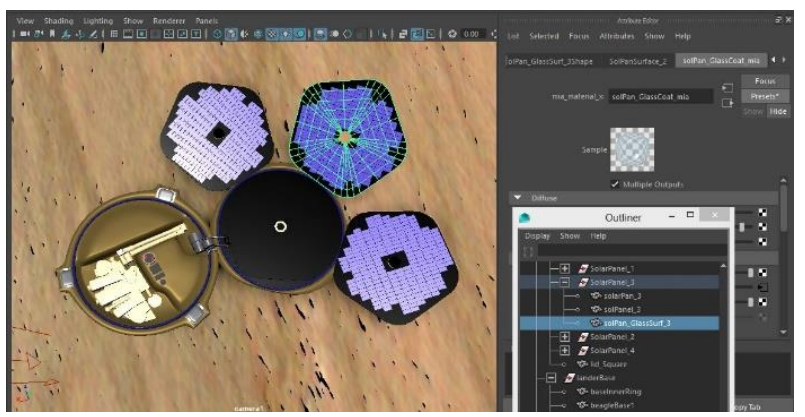

Figure 4: MIA materials (solar panel glass cover)

Due to the time constraints of the project, it was decided that the practical experimentation would focus on a comparison between the sun angles, reproduced in 3D renders and the HiRISE satellite images depicting three different lighting scenarios, corresponding respectively to Sun azimuth illumination angles of 136.68, 139.76 and 203.76 degrees.

The relevant lighting conditions were replicated in the software by setting the rotation attribute of the light to match these degrees precisely.

ESP_030908_1915_RED
Beagle 2 Hardware: Lander (7909,46014)
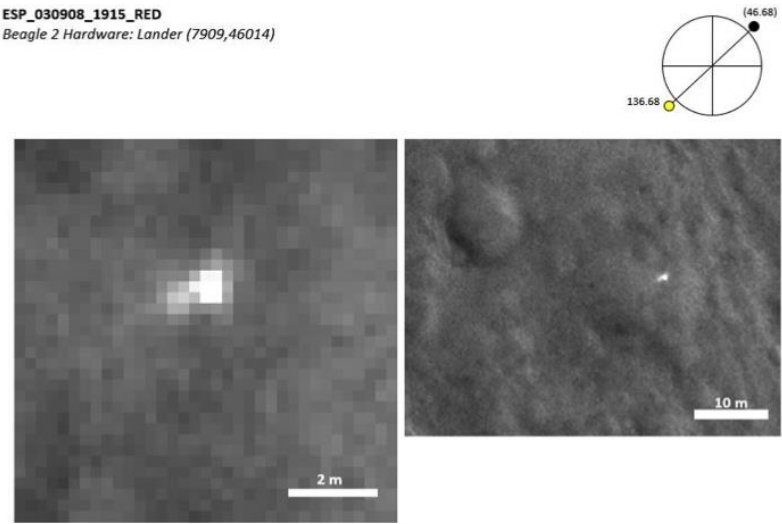

Figure 5: - Beagle 2 coordinates and solar longitude (real satellite image, sun angle 136.68)

\subsection{Cameras and rendering}

Apart from the default cameras in Autodesk Maya, two virtual cameras were created for the purpose of exporting 3D renders for comparison to the HiRISE images. The settings of both cameras were adjusted to simulate the resolution and view point of NASA's Mars Reconnaissance Orbiter.

After attempting to also replicate the approximate distance of the MRO to the target site by inputting a relevant value for the Translate $Y$ attribute of one of the cameras, it became apparent that the software posed certain limitations in this respect. The experiment resulted in both the model of the lander and the terrain disappearing from the viewport, and the problem had to be resolved by positioning the camera closer to the object - the Translate $Y$ attribute's value was decreased considerably, until the size of the probe visible on screen matched the size of the actual Beagle 2 on the HiRISE images in terms of pixels. The other camera shared the same viewpoint but was positioned even closer to the lander to allow the export of close-up renders, presenting a more detailed view of the model.

The views from both virtual cameras were exported simultaneously to provide renders matching the view from the camera of the Mars Reconnaissance Orbiter, as well as a corresponding close-up of lander.

The HiRISE images needed for the comparison were downloaded from the Lunar \& Planetary Laboratory. Due to the JP2 (JPEG2000) file format of the original images and the limited number of software applications capable of working with this format, the HiView data explorer and image viewing application was utilised. While the large native dimensions and file size (ranging from $500 \mathrm{Mb}$ to near $1 \mathrm{~Gb}$ ) of the HiRISE images would have posed a challenge in terms of their use, the HiView explorer made it possible for a specified area in the probe's immediate vicinity to be saved in a different format and used with an image editing application for further enhancement of any particular region of interest at full resolution.

After configuring Autodesk Maya's Render Settings to match the satellite images' resolution and identified region around the probe, a series of renders of the lander's model were exported. Extensive experimentation was carried out to compare the HiRISE images to the software renders depicting different sun angles (136.68, 139.76 and 203.76 degrees) and orientations for the Beagle 2 model, including a slight tilt of the object and all possible configurations of the solar panels deployment. All scenarios with respect to the possible number of open panels were explored (1, 2, 3 and 4).

The investigation of panels' opening configuration in combination with different orientation of the lander and its tilt with respect to the surface of Mars proved that the number of different scenarios could be almost interminable. Due to the strict time constrains of the project and the average amount of time the software required to export each render, there were concerns regarding the successful outcome of the research, especially as the initial comparison of software renders to satellite images failed to demonstrate possible congruency between them. 
At this critical point the assistance of Dr Mark Sims was instrumental in terms of narrowing down the possible orientation of the probe to a couple of viable scenarios and determining a limit for the maximum angle of the lander with respect to the surface of Mars. During a careful inspection of the software renders compared to the satellite images, Dr Sims advised that the brightest reflection was most likely from the second solar panel, or the lander's base, at a possible tilt of up to 20 degrees.



Figure 6: Lander configuration at a sun angle of 136.68 degrees with 3 panels open (3D render)

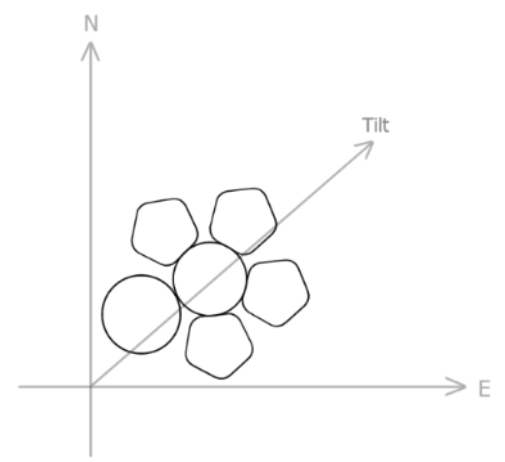

Figure 7: Lander orientation diagram: top

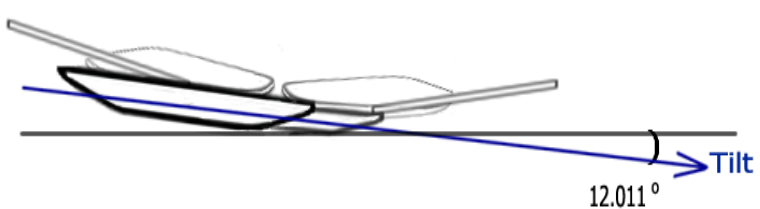

Figure 8: Lander orientation diagram: cross-section

This estimation pointed to a more particular orientation of the probe and a consequent series of renders were exported where the scrutiny of software images proved to be consistent with the base being located 'south-west' of the lid and tilted up at approximately 12 degrees towards the 'northeast'.

The visual comparison of the selected for the simulation three different lighting sun angles in rendered images proved to be congruent to the HiRISE data. As a visual comparison would have been inadequate in terms of presenting conclusive scientific results), the quality of final renders, exported from Autodesk Maya, was degraded (pixelised), so the number of their pixels matched precisely the ones from the satellite images.

The pixelisation was achieved through the utilisation of the Mosaic Filter in Photoshop. The 3D renders and the satellite images were cropped at the same number of pixels and exact position of the lander.

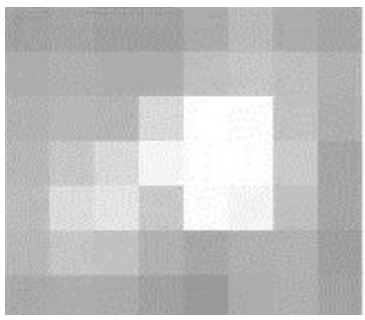

(a)

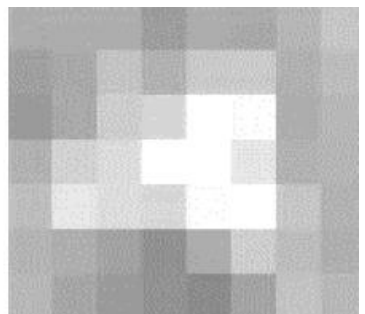

(b)
Figure 9: Pixel correlation of the lander at a sun angle of 136.68 degrees with 3 panels open. The respective images show (a) satellite, cropped, and

(b) 3D render, cropped.

\section{RESULTS}

"Focusing on the area local to the target, the satellite and simulation images were normalised to give a maximum pixel value of 1 . The similarity of the images was then compared using three approaches: correlation, the mean pixel value of a subtracted image (simulation image - satellite image) and finally the mean pixel value of a ratioed image (satellite image/simulation image)" (Clemmet 2017).

The initial image correlation test was undertaken with the ImageJ software and the mathematical representation of pixel intensity values for the image pairs (3D renders and satellite images), used for the experiment was outputted in Microsoft Excel. All image renders and correlation results were then sent to Dr Mark Sims and his team of imaging specialists for further analysis.

The final image analysis (including spectral analysis) carried out by scientists from the Leicester University, was performed with custom software written in $\mathrm{R}$ and can easily be repeated or expanded to include additional images. Three satellite images, each from a different angle, were 
compared with 11 simulated images - the results of a simulation of Beagle with 1, 2, 3, or 4 panels fully opened and 4 panels open at an angle of $130^{\circ}$ (Table 1). In some cases, multiple tests were run for each panel configuration with the simulation image down-sampled to the same number of pixels as the satellite image in slightly different ways these have not been separated out in the results.

For each background threshold, there are three possible measures of similarity to the satellite image (correlation, difference, ratio), although these measures are somewhat related, they do not always give identical results. The table below summarises the number of times each panel configuration was considered to be the best match, across all tests (nine tests total). Also given is the average number of pixels included within the satellite area in each case where that panel configuration was considered the best match.

Table 1: Simulation results

\begin{tabular}{|l|l|l|l|l|}
\hline \multirow{3}{*}{$\begin{array}{l}\text { Panel } \\
\text { Configuration }\end{array}$} & \multicolumn{2}{l|}{$\mu_{\mathrm{b}}+3 \sigma_{\mathrm{b}}$} & \multicolumn{2}{l|}{$\mu_{\mathrm{b}}+5 \sigma_{\mathrm{b}}$} \\
\cline { 2 - 5 } & $\begin{array}{l}\text { No of } \\
\text { times } \\
\text { most } \\
\text { similar }\end{array}$ & $\begin{array}{l}\text { Mean } \\
\text { No of } \\
\text { pixels }\end{array}$ & $\begin{array}{l}\text { Number } \\
\text { of times } \\
\text { most } \\
\text { similar }\end{array}$ & $\begin{array}{l}\text { Mean } \\
\text { No of } \\
\text { pixels }\end{array}$ \\
\hline 1 & 1 & 12 & 2 & 8 \\
\hline 2 & 0 & - & 0 & - \\
\hline 3 & 4 & 20.25 & 3 & 18 \\
\hline 4 & 1 & 12 & 0 & - \\
\hline 4 at $130^{\circ}$ & 3 & 14 & 4 & 10.25 \\
\hline
\end{tabular}

The most common results were the 3 panel and 4 panels at $130^{\circ}$ configurations. If number of pixels within satellite area is used as a proxy for image quality, and so for accuracy of results, then the 3 panels configuration can be considered the best match. It may be that the true configuration is somewhere between these configurations.

In Dr Jim Clemmet's expert opinion, the 136 and 139 degrees illumination cases do not exclude the possibility of the 4th panel deployment, as it is completely possible that the panel is not reflecting - furthermore, the 203 degrees case provides strong evidence of the panel 4 deployed to 130 degrees. HiRISE images with similar or better illumination angles would be of strong benefit to demonstrate consistency and establish the actual Beagle 2 configuration with a higher degree of certainty. Based on the image analysis results, $\mathrm{Dr}$ Clemmet's paper Beagle 2 on Mars - the Discovery Assessed, provides a detailed account of the successes and mission loss possible scenarios. The paper was recently published in the Journal of the British Interplanetary Society (Clemmet 2017).

\section{CONCLUSIONS, DISCUSSION AND FUTURE PLANS}

The collaboration between De Montfort University and the University of Leicester succeeded in bringing to light some aspects of the fate of Beagle 2 after being ejected from the ESA's orbiter spacecraft Mars Express. As Dr Mark Sims stated in an interview, which received a large publicity in both UK and international media and news sources, "[t]his work (further) confirms that the Entry, Descent and Landing (EDL) sequence for Beagle 2 worked as expected and the lander did successfully touchdown on Mars on Christmas Day 2003" (Sims 2003). The primary practical research also managed to reach the "exciting conclusion that Beagle 2 did not crash, but landed and probably deployed most of its panels" (Higgett 2016), and also identified the possible configurations of the lander having deployed 3 panels, or 4 panels at $130^{\circ}$

With regards to the initial findings of the project, $\mathrm{Dr}$ Sims stated, "[t] his unique University collaboration between space scientists and digital designers allowed this reflection analysis concept to be put into practice and tested, and ultimately produce these exciting results" (Sims 2016).

Future plans, regarding the continuation of the project involve the employment of 3D software not only for the purpose of finding answers to specific questions, regarding the fate of Beagle 2, but also to establish practical evidence regarding the degree of suitability of Dr Sim's 'reflection analysis' technique. While initial experimentation with the technique proved invaluable in identifying the object on the surface of Mars as Beagle 2 and revealing its possible configuration, it also returned indications that the 'reflection analysis' might have the full potential not only to determine the specific causes of Beagle 2's failure beyond doubt, but to re-write existing scientific methods in a much broader scope. The 'reflection analysis' could serve the purposes of testing and evaluating the usability of a wide range of technical devices and equipment in a virtual environment, simulating the physical properties of objects and natural dynamic forces, such as gravity, wind, turbulence, pressure.

Further work on the practical research would attempt to pinpoint the causes for the Beagle 2 mission's lack of success, as understanding failure modes is critical for the evolution in spacecraft design. Understanding the necessary changes, stemming from the lessons learnt, would provide future missions with a key direction, and allow them to avoid similar problems. From a more unambiguous point of view, the continuation of the project would enable the Beagle 2 team to estimate how close they were to success and provide 
closure on what looked like an unknown failure and total loss back in 2003.

The practical work with 3D software would also complement other experimental techniques, such as super-resolution imaging as conducted by Professor Jan-Peter Muller and his team at University College London, announced in April 2016, which is based on stacking and matching images, captured by a satellite, to reveal objects at a greater resolution. The technique, which recently became known as Super-Resolution Restoration (SRR), was utilised in a similar practical experiment, focusing on discovering Beagle 2 on the surface of Mars. The project has been carried out independently from the collaboration between De Montfort University and the University of Leicester, but arrived at the same conclusions.

\section{REFERENCES}

Astronomy Now (2016).

https://astronomynow.com/2016/11/11/did-a-failedsolar-panel-block-beagle-2s-antenna/

(retrieved 23 November 2016).

Autodesk (2014).

https://knowledge.autodesk.com/support/maya/lear n-explore/caas/CloudHelp/cloudhelp/2015/ENU/Ma ya/files/GUID-E5BB5C0C-CB68-4DF1-AC46EDA62BD95083-htm.html (retrieved 3 March 2016).

Autodesk (2016).

https://knowledge.autodesk.com/support/maya/lear n-explore/caas/CloudHelp/cloudhelp/2016/ENU/Ma ya/files/GUID-F5429279-8EDB-449C-9E8D4B5C0210757D-htm.html (retrieved 20 February 2016).

Beagle 2 (2016). http://beagle2.our.dmu.ac.uk (retrieved 18 November 2016).

BBC News (2016).

http://www.bbc.co.uk/news/science-environment$\underline{37940445}$ (retrieved 9 December 2016).
Clemmet, J. (2017) Beagle 2 on Mars - the Discovery Assessed. Journal of the British Interplanetary Society, 70(8):262-278.

HiRise (2016) Lunar \& Planetary Laboratory. https://www.uahirise.org/results.php?keyword=bea gle+2\&order=release date\&submit=Search (retrieved 7 March 2016).

O'Connor, J. (2010) Mastering mental ray: Rendering Techniques for $3 D$ and CAD Professionals. John Wiley \& Sons, Indianapolis.

Palamar, T. (2010) Maya Studio Projects: Dynamics. John Wiley \& Sons, Indianapolis.

Pillinger, C. (2003) The Guide to Beagle 2. Alden Group, Oxford.

Popat, S., and Palmer, S. (2008) Creating Common Ground: Dialogues between performance and digital technologies, International Journal of Performance Arts \& Digital Media, 1(1):47-65.

Sky News (2016). http://news.sky.com/story/beagle-2-probe-did-notcrash-land-on-mars-new-images-show-10652864 (retrieved 12 December 2016).

Tang, M. (2014) Parametric Building Design Using Autodesk Maya. Routledge, London.

Tao, Y., and Muller, J-P. (2015) A Novel Method for Surface Exploration: Super-resolution restoration of Mars repeat-pass orbital imagery. Planetary and Space Science, 121(2):103-114.

UCL News (2016).

https://www.ucl.ac.uk/news/newsarticles/0416/260416-Mars-images (retrieved 15 December 2016).

UK Space Agency (2016). https://www.gov.uk/government/news/uk-ledbeagle-2-lander-found-on-mars (retrieved 5 November 2016).

University of Leicester (2016). https://www.sciencedaily.com/releases/2016/11/16 1110212712.htm (retrieved 18 November 2016). 